\title{
David M. Knipe, Vedic Voices. Intimate Narratives of a Living Andhra Tradition
}

Oxford-New York, Oxford University Press, 2015, 368 p., cartes, photographies, glossaire, index, bibliographie.

\section{Catherine Clémentin-Ojha}

\section{(2) OpenEdition}

Journals

Édition électronique

URL : http://journals.openedition.org/assr/28263

DOI : $10.4000 /$ assr.28263

ISSN : $1777-5825$

Éditeur

Éditions de l'EHESS

Édition imprimée

Date de publication : 31 décembre 2016

Pagination : 335

ISSN : 0335-5985

\section{Référence électronique}

Catherine Clémentin-Ojha, « David M. Knipe, Vedic Voices. Intimate Narratives of a Living Andhra Tradition », Archives de sciences sociales des religions [En ligne], 176 | octobre-décembre 2016, mis en ligne le 18 juillet 2017, consulté le 24 septembre 2020. URL : http://journals.openedition.org/assr/ 28263 ; DOI : https://doi.org/10.4000/assr.28263

Ce document a été généré automatiquement le 24 septembre 2020.

(c) Archives de sciences sociales des religions 


\section{David M. Knipe, Vedic Voices. Intimate Narratives of a Living Andhra Tradition}

Oxford-New York, Oxford University Press, 2015, 368 p., cartes, photographies, glossaire, index, bibliographie.

\section{Catherine Clémentin-Ojha}

\section{RÉFÉRENCE}

David M. Knipe, Vedic Voices. Intimate Narratives of a Living Andhra Tradition, OxfordNew York, Oxford University Press, 2015, 368 p.

1 Voilà un ouvrage qui allie la connaissance des textes du Veda, acquise par des études d'histoire intellectuelle, et un travail de type ethnographique mené au cours d'une enquête de terrain. S'agissant du Veda, le mariage de ces deux compétences est inattendu. Composé en sanskrit entre le $\mathrm{xv}^{\mathrm{e}}$ et le $\mathrm{v}^{\mathrm{e}}$ siècle avant notre ère, le Veda, vaste corpus d'hymnes, de récits, d'exégèse et de spéculations, se concentre sur la célébration du sacrifice. Il prescrit et décrit diverses pratiques rituelles. Toutefois celles-ci ont presque entièrement disparu. Du Veda, il ne subsiste qu'un ensemble de textes - au demeurant de toute première importance pour l'économie de l'hindouisme. Le spécialiste de la « religion védique » est un philologue. Si David M. Knipe, professeur émérite du département de langues et cultures de l'Asie et du département des études religieuses de l'université de Wisconsin, s'est aussi fait ethnologue, c'est pour présenter une petite communauté de prêtres qui perpétue les rites sacrificiels védiques et s'efforce de vivre en respectant les règles prescrites par les anciens textes. Ces personnes extraordinaires sont des brahmanes védiques (vaidika, en sanskrit) au sens premier de l'adjectif et non dans celui rencontré couramment dans l'Inde contemporaine, où, en ces temps de revivalisme hindou, on exploite le Veda pour donner un cachet traditionnel à toutes sortes de pratiques, de l'astrologie à l'écologie 
en passant par l'architecture, comme l'a récemment montré un colloque organisé au Centre d'études de l'Inde et de l'Asie du Sud («In the Name of the Veda », 14-15 mai 2014).

Les brahmanes en question vivent dans la riche région agricole du delta de la Godavari en Andhra Pradesh (Inde du Sud). Entre 1980 et 2015, l'auteur a fréquenté une dizaine de leurs familles et conduit une centaine d'entretiens avec l'aide d'assistants parlant la langue telugu. Il nous fait pénétrer dans le quotidien de quatre générations en organisant son ouvrage autour des récits de vie qu'il a ainsi recueillis ( $A$ A Selection of Ahitagni and Other Veda Pandits in Agrahara, Villages and Towns »- la liste des personnes mentionnées se trouve p. 139). Dans deux chapitres précédents, il présente l'histoire et la géographie de la région indienne concernée par l'enquête ("The Godavari Delta ») et donne quelques repères pour suivre le déroulement d'une carrière de pandit védique ("Vedamlo, 'Living in the Veda'»). Dans les trois chapitres subséquents, il reprend les informations significatives des récits de vie selon une grille thématique ( Becoming a Veda »; « Becoming a Householder »; « Becoming Agni »). Son septième et dernier chapitre évoque les changements contemporains ("Becoming 'modern'»).

3 Les brahmanes qui « vivent dans le Veda » (vedamlo, en telugu) constituent un monde à part non seulement de la majorité des hindous, mais aussi des autres brahmanes non védiques, qui, selon eux, ont abandonné leur brahmanité (brahmatva) et sont devenus " mondains " (laukika). Ils ne forment pas une catégorie homogène cependant, mais se divisent entre ceux qui célèbrent les rites védiques domestiques et solennels et ceux qui ne célèbrent que les premiers. Ces deux catégories sont toutefois intersécantes puisqu'il arrive qu'un fils ne reprenne pas toutes les pratiques rituelles de son père sans pour autant cesser d'être un brahmane védique.

4 La condition de brahmane védique résulte de la naissance et de l'éducation. Tout commence par l'initiation brahmanique (upanayana) du jeune garçon, né dans une famille de brahmanes védiques, suivie de peu par son mariage, que ses parents organisent avec une fillette de la même sous-caste de brahmanes védiques que la leur (mais d'une lignée distincte d'ancêtres, gotra). Pendant les huit à douze ans qui suivent, le garçon apprend sous la direction d'un aîné de sa famille (souvent le père de son père) la recension du Veda de sa tradition familiale (en l'occurrence celle de la Taittirìya Śākhā du Kṛ̣ṇa Yajur Veda). La quantité de texte ainsi mémorisé est phénoménale : elle correspond à 2000 pages de texte imprimé (p. 34). Au sortir de ces études, son épouse et lui commencent leur vie commune en allumant leur propre feu domestique. Dans ce feu domestique, tous les brahmanes védiques doivent faire des libations (homa) biquotidiennes. En plus de cela, certains d'entre eux maintiennent les trois feux du rituel védique solennel (śrauta) dans une pièce spéciale de leur maison (plan p. 42) : ce sont les ahitāgni («[ceux] qui ont allumé le feu»). L'attention de l'auteur se porte surtout sur cette catégorie qui, à l'heure actuelle, compterait quelque 600 membres en Inde et au Népal, dont près d'un tiers vivrait dans le seul Andra Pradesh (p. 41). Parce qu'ils doivent faire deux fois par jour le rite de l'agnihotra, consistant en libations de lait chauffé, les ahitagni sont aussi appelés agnihotrin.

Outre qu'il contient de nombreux détails techniques sur l'apprentissage et la célébration des rites sacrificiels védiques, qui font parfois penser au lecteur qui lit un manuel d'études indiennes classiques, l'ouvrage fourmille d'observations très intéressantes sur la façon dont ces hindous ultra-orthodoxes vivent dans la société indienne d'aujourd'hui et se représentent leur monde. 
On apprend ainsi que seuls quelques-uns poursuivent leurs études pour devenir pandit, en se spécialisant dans des études de "philosophie» (vedānta, mīmāmisā). La plupart sont des prêtres domestiques (rituel smārta) ou des récitants professionnels du Veda dans les temples où l'on adore les divinités du panthéon hindou. Si pour beaucoup être «a Vedic voice for hire» (p. 93) est un moyen acceptable de gagner sa vie, à condition de ne pas frayer avec les officiants du culte divin (pújā), pour d'autres, d'une génération plus ancienne notamment, c'est "vendre le Veda" (veda-vikraya), le plus grand des crimes. Certaines familles vivent des revenus de terres agricoles qui ont été données à leurs ancêtres par de riches patrons. Certaines bénéficient du patronage étatique de la "culture védique», qui a remplacé le patronage des cours royales, et attribue des pensions et des prix spéciaux pour récompenser les brahmanes se distinguant par leur savoir.

7 Cette galerie de portraits intimes, rendus plus vivants encore grâce aux photographies, souvent touchantes, prises par l'auteur, révèle aussi une vision du monde. Toute la vie de ces brahmanes tourne autour du Veda et de la préservation de son texte et des rites qu'il enjoint. Comme on peut s'y attendre, lesdits brahmanes se représentent le Veda comme un corpus de textes entièrement soustrait à l'histoire, qui n'est pas inscrit dans le temps qui passe, n'a pas d'auteur humain, est éternel. Il est aussi unitaire. Les brahmanes védiques ne peuvent entendre que les textes qui composent le Veda n'ont pas tous la même chronologie, ni que les upanisad ont représenté un changement profond dans la vision du monde védique, comme l'enseigne la science indianiste. Pour autant, ils ne sont pas dénués de toute conscience historique. Ils ont une parfaite connaissance de leur lignée; certains peuvent même nommer leurs ancêtres sur les huit générations qui les ont précédés (p. 146). S'il en est ainsi, c'est que chacun est particulièrement conscient de représenter un chainon dans la transmission du Veda, et extrêmement préoccupé de l'éventuelle interruption de celle-ci, toujours possible. Car tout brahmane védique, et plus particulièrement encore les ahitagni, est habité par trois ambitions: absorber le Veda, maîtriser le complexe savoir rituel nécessaire à la célébration des rites sacrificiels et préparer ses descendants à suivre la même voie. Or, comme le montrent la plupart des récits de vie, l'univers des brahmanes védiques est menacé. La transmission de leur style de vie est en danger, parce que les temps changent et que l'appel de nouveaux métiers est irrésistible dans bien des familles. Toutefois, quelques lignées sont plus prometteuses. En outre, il n'est interdit à personne de réactiver une ancienne tradition familiale, ni à tel ou tel fils aîné d'installer les trois feux au mitan de sa vie, après avoir considéré leur entretien trop exigeant dans ses jeunes années. Ce qui au passage montre que dans la réalité la pratique de l'agnihotra n'est pas la tradition ininterrompue qu'on prétend, mais peut avoir été momentanément suspendue, et donc être présentée comme telle. Les textes védiques prévoient d'ailleurs les rites à accomplir pour les arrêts et les reprises, ceux occasionnés par les voyages notamment.

Parmi les conditions à remplir pour assurer la pérennité d'une lignée de transmission figurent les règles du mariage. En parler conduit à considérer la situation de la femme chez les brahmanes védiques. L'auteur ne laisse pas le lecteur l'oublier, même s'il n'a pas eu le même accès aux femmes qu'aux hommes, et a dépendu de l'enquête conduite par une collègue indienne (originaire du milieu des brahmanes védiques) pour s'informer. Deux ou trois figures féminines bien individualisées émergent de son ouvrage. Dans l'exécution des rites, qui nécessite la récitation des textes du Veda 
mémorisés pendant les études, l'officiant n'est pas seul, mais nécessairement accompagné de son épouse. Devenu veuf, il perd ses prérogatives rituelles et ne les recouvre qu'une fois remarié. Certes les femmes ne célèbrent pas elles-mêmes les rites, mais leur présence auprès des feux est requise ; elles tiennent un rôle d'assistante, que leur mari leur enseigne. Comme partenaires rituelles, il leur incombe d'assurer la pureté requise pour la célébration des rites (madi): pureté corporelle, pureté des vêtements, pureté des lieux, pureté de la nourriture. Comme génitrices de futurs brahmanes védiques aussi, elles doivent être pures. Pour garantir la pureté de leur lignée, les brahmanes en question épousent des filles prépubères (rajasvalā-púrva) nées elles-mêmes d'une femme dont le mariage avait été célébré avant sa puberté (p. 162). Ils passent donc outre la loi indienne qui, depuis 1955, interdit les mariages d'enfants (p. 165). Pourtant tout le monde ne s'y résout pas. C'est parce qu'il a paru impossible à un veuf de 61 ans d'épouser en secondes noces une fille prépubère qu'il a préféré mettre un terme à sa vie rituelle (p.123) - choix déchirant. Outre le mariage dans la sous-caste, commun à l'ensemble du monde hindou, on observe aussi dans ce milieu le mariage préférentiel avec la cousine ou le cousin croisés - un garçon épouse la fille du frère de la mère, une fille le fils de la sœur de son père. Signalons que fréquemment observée en Inde du Sud, cette règle, qui empêche la dispersion de l'héritage tout en garantissant la pureté de la lignée, est interdite en Inde du Nord.

9 La pérennité d'une lignée de brahmanes védiques dépend aussi de la capacité des fils à prendre la relève de leur père. Deux causes d'interruption des rites solennels retiennent plus particulièrement l'attention. L'une résulte de la règle qui interdit à un frère cadet d'installer les trois feux avant l'aîné de la lignée ; l'auteur donne quelques exemples de lignées d'ahitāgni interrompues pour cette raison (p. 77, 118). L'autre cause d'interruption résulte de ce qu'il faut considérer comme une sorte de conversion. L'illustre le cas de ce jeune homme devenu dévot de Śiva et ayant adopté la religion de la bhakti - religion que les brahmanes védiques considèrent avec quelque mépris (p. 77). Cet épisode, qui témoigne de la difficulté de ces brahmanes à maintenir la tradition védique pure de toute contamination hindoue, rappelle la nature contradictoire des liens entre l'hindouisme et le védisme. L'hindouisme sort du védisme : c'est-à-dire qu'il en possède maints traits, mais cela signifie aussi qu'il s'en écarte. Sans doute le trait le plus spécifiquement védique que l'ouvrage met en lumière est-il l'obsession de ces brahmanes pour l'exactitude des rites : le mal réside dans l'erreur.

Les plus belles pages du livre portent sur Agni, le feu et la divinité qui l'incarne. L'auteur sait faire partager sa fascination pour ces hommes qui, dans l'Inde contemporaine, maintiennent une tradition de plus de trois millénaires en installant Agni, en l'alimentant avec des brindilles, et en passant des milliers d'heures de leur vie à lui réciter les mantras du Veda mémorisés pendant leurs études, à inhaler sa fumée, à regarder danser ses flammes. Agni est le seigneur de leur maison et de leur famille. Tout au long de leur vie, cette forme élémentaire du sacré (p.49) les absorbe entièrement. Et après leur mort, livrés au feu «qui dévore la chair» (kravyād), transformés en oblation, ces brahmanes deviennent eux-mêmes Agni. 